Clinica Circingica

\section{H. PYLORI E CÂNCER GÁSTRICO}

McColl e El-Omar, do Departamento de Medicina da Universidade de Glasgow, relatam que oH. pylori atualmente é reconhecido como um co-fator importante na etiologia do câncer gástrico. Os tumores do tipo histológico-intestinal se desenvolvem de uma forma complexa e multifatorial; inicialmente ocorre a progressão de gastrite superficial para a gastrite atrófica com metaplasia intestinal e hipocloridria. Esta alteração da mucosa gástrica pode evoluir para displasia e câncer. Muitos co-fatores estão envolvidos nesta progressão, incluindo linhagem do $H$. pylori, fatores genéticos do doente, interleucina-I e fatores ambientais como o fumo e a dieta. A colonização intestinal por helmintos também pode alterar a imunidade e resposta inflamatória ao $H$. pylori. Como conseqüência, a prevenção da infecção ou a erradicação precoce desta bactéria reduz a incidência deste tipo de tumor.

\section{Comentário}

Os mecanismos que implicam o $\mathrm{H}$. pylori na etiopatogenia do carcinoma gástrico ainda não estão devidamente esclarecidos e são discutidos por vários autores, mas a infecçãa por esta bactéria tem sido relacionada com 0 linfoma gástrico e, embora existam controvérsias, também ao adenocarcinoma. Possivelmente existem diferentes graus de virulência entre as cepas da bactéria, que rompe a barreira da mucosa do estômago através de toxinas, enzimas e mediadores da inflamação, provocando dano celular epitelial, alteração do fluxo sangüíneo na mucosa, alteração do muco e da liberação de gastrina. A liberação de gastrina após as refeições está aumentada nos infectados pelo $\mathrm{H}$. pylori e retorna ao normal após a sua erradicação.

As cepas mais virulentas têm maior motilidade e produzem enzimas adaptativas, tais como urease, lipase, oxidase, mucinase, catalase, protease e fosfolipase, assim como fatores quimiotáticos para os neutrófilos e monócitos, citocinas, fatores de adesão e proteína inibitória da produção de ácido.

Embora muitos aspectos a respeito desta bactéria e a virulência de suas cepas ainda estão por ser elucidados, a efetividade do tratamento e a melhora clínica dos doentes ulcerosos e portadores de dispepsia funcional tratados, além de sua associação com o câncer gástrico, justificam seu tratamento sempre.

\section{Pedro luiz Squilacci leme} Caio Romeiro Bove Rodrigo Altenfelder Silva

\section{Referências}

I. McColl KE, El-Omar E. How does H. Pylori infection cause gastric cancer? Keio J Med 2002; 5I(Suppl 2):53-6.

2. Safatle-Ribeiro AV, Ribeiro U Jr, Lopasso FP, Deutsch C, Mucerino D, Arab-Fadul R, et al. Fatores de risco para o câncer gástrico. In: Gama-Rodrigues J], Lopasso FP, Del Grande JC, Safatle NF, Bresciani C, Malheiros CA, et al., editores. Câncer do estômago. Aspectos atuais do diagnóstico e tratamento. São Paulo: Andrei; 2002.p.29-58.

3. Rahal F. Doença ulcerosa. In: Rahal F, Pereira V, Malheiros CA, Rodrigues FCM, Gonçalves AJ, editores. Condutas normativas - Departamento de Cirurgia da Faculdade de Ciências Médicas da Santa Casa de São Paulo. $9^{2}$ ed. São Paulo; 1998. p.10-27.

\section{Emergência e Medicina Tutensiva APOPTOSE E DISFUNÇÃO ORGÂNICA INDUZIDA PELA VENTILAÇÃO PULMONAR MECÂNICA NA SDRA}

A ventilação pulmonar mecânica pode piorar a lesão pulmonar preexistente e produzir uma lesão pulmonar induzida pelo ventilador. Entretanto, não está claro o mecanismo preciso através do qual a ventilação pode ocasionar esta lesão pulmonar e, indiretamente, determinar a disfunção de órgãos à distância.

Recentemente, uma pesquisa médica de interpretação examinou a hipótese de que a estratégia ventilatória agressiva possa determinar a apoptose de células epiteliais em diversos órgãos, ocasionando disfunção orgânica. Em um modelo experimental de lesão pulmonar com aspiração, Imai Y et al, 2003', analisaram 24 coelhos em que se utilizo duas estratégias ventilatórias: agressiva e não-agressiva, determinando os marcadores bioquímicos de disfunção hepática e renal e a apoptose em determinados órgãos. Foi também medida in vitro a apoptose das células epiteliais do túbulo renal LLC-RKI, além da correlação da creatinina plasmática e do ligante FAS solúvel de amostras plasmáticas de pacientes incluídos em uma pesquisa prévia ${ }^{2}$ em que se examinou a estratégia de proteção pulmonar comparativamente a um grupo controle. A estratégia ventilatória não-protetora aumentou as taxas de apoptose das células epiteliais no rim, no intestino delgado e ocasionou in vivo o aumento dos marcadores bioquímicos indicativos de disfunção renal. Houve também uma correlação significante entre as alterações do ligante FAS solúvel e a creatinina nos pacientes com SDRA ( $R=0.64$, $P=.002)$. Estes achados podem explicar parcialmente a alta taxa de síndrome de disfunção de múltiplos órgãos observada nos pacientes com SDRA e a diminuição de morbidade e mortalidade nos pacientes tratados com a estratégia de ventilação protetora.

\section{Comentário}

Esta é uma pesquisa de bancada experimental que nos permite uma interface clínica importante com uma ampliação do conhecimento médico e uma possibilidade de melhora dos cuidados do paciente. Este trabalho amplia os dados obtidos pelas pesquisas clínicas, demonstrando que a utilização de estratégias ventilatórias protetoras está associada com uma diminuição dos níveis de citocinas e quimoquinas $^{2}$, diminuição da disfunção orgânica ${ }^{3}$ e da mortalidade em pacientes com SDRA, sugerindo que os mediadores que aumentam a apoptose das células epiteliais dos órgãos podem ser objetivos terapêuticos razoáveis a serem atingidos para alterar a síndrome de disfunção de múltiplos órgãos nos pacientes com SDRA.

\section{Werther Brunow de Carvalho}

Flávia Vanesca felix leão

Referências

I. Imai Y, Parodo J, Kajikawa O, de Perrot M, Fischer S, Edwards V, et al. Injurious mechanical ventilation and end-organ epithelial cell apoptosis and organ dysfunction in an experi- 
mental model of acute respiratory distress syndrome. JAMA 2003; 289(I6):2104-12.

2. Ranieri VM, Suter PM, Tortorella C, De Tullio R, DayerJM, Brienza A, et al. Slutsky AS. Effect of mechanical ventilation on inflammatory mediators in patients with acute respiratory distress syndrome: a randomized controlled trial. JAMA 1999; 282(I):54-6I.

3. Ranieri VM, Giunta F, Suter PM, Slutsky AS. Mechanical ventilation as a mediator of multisystem organ failure in acute respiratory distress syndrome. JAMA. 2000 Jul 5;284(I):43-4.

\section{Ginecologia \\ VOZ E MUDANÇAS VOCAIS APÓS A MENOPAUSA}

Boulet \& Oddens $(1996)^{2}$ relatam que após a menopausa ocorrem mudanças na voz, decorrentes do impacto do hipoestrogenismo sobre a laringe e que foram confirmadas pelos esfregaços das pregas vocais que se mostraram atróficos e semelhantes àqueles do colo uterino (Abitbol \& Abitbol, 1998)'. Esse último estudo, realizado em 100 mulheres após a menopausa, constatou expressivas alterações laríngeas e vocais.

\section{Comentário}

Nos estudos acima, descritos e realizados com cantoras na Bélgica, Holanda, Áustria e com profissionais da voz na França, apesar das mulheres revelarem conhecimento da importância da emissão vocal em suas atividades e terem refinamento auditivo para perceberem as alterações, um significativo número delas não procurou especialistas com o objetivo preventivo de retardar ou amenizar os agravos da redução hormonal do climatério e do envelhecimento sobre o processo vocal.

Em investigação qualitativa realizada por nós na cidade de Curitiba ${ }^{3}$ - Paraná, constatamos que a produção vocal é utilizada na comunicação para 0 estabelecimento de critérios relacionais e autoreflexão na vida cotidiana. 0 processo vocal foi considerado motivo de atenção pessoal para uma parcela expressiva de mulheres após a menopausa, não profissionais da voz; o estudo evidenciou, também, que as alterações vocais estabelecem conflitos ou contradições na constituição da nova identidade, sugerindo, por isso, que os fatores biopsicossociais devam sempre ser considerados como importantes elementos de repercussão sobre a voz (Machado, Aldrighi \& Yunes*, 2003) ${ }^{3}$.

Lindholm (1997), ao investigar a intensidade vocal na fala espontânea e na leitura, em mulheres após a menopausa que não utilizavam a voz profissionalmente, averiguou que a terapia de reposição hormonal atua como importante fator protetor aos agravos da voz no climatério. Abitbol \& Abitbol (1998)', por outro lado, indicam a reeducação vocal, caso a sintomatologia persista após três meses da terapêutica medicamentosa.

Do exposto, é importante alertar os profissionais de saúde que atendem mulheres no climatério, especialmente aquelas que utilizam a voz como principal instrumento de trabalho, objetivando não só manter o desempenho adequado na ocupação exercida, mas também a qualidade de vida após a menopausa.

Maria Aparecida M. de P. Machado

José Mendes Aldrighi JoÃo Yunes*

Referências

* iN MEMORIAN

I. Abtibol J, Abtibol B. Voix et menopause: crepuscule des divas Contracept Fertil Sex 1998; 26:649-55.

2. Boulet MJ, Oddens BJ. Female voice changes around and after the menopause-an initial investigation. Maturitas 1996:23: 15-21. 3. Machado MAMP. Tese de Doutorado a ser defendida no Departamento de Saúde Materno-Infantil da Faculdade de Saúde Pública da Universidade de São Paulo, 2003.

4. Lindholm P, Vilkman E, Raudaskoski T, Suvanto-Luukkonen E, Kauppila A. The effect of postmenopause and postmenopousal HRT on measured voice and vocal symptons. Maturitas 1997; 28:47-53.

\section{Medicina Baseada em Euidencias MANOBRAS DE RECRUTAMENTO PULMONAR NA SDRA}

O tratamento da síndrome do desconforto respiratório agudo (SDRA) tem sido motivo de importantes controvérsias na terapia intensiva. A mortalidade da SDRA oscila em torno de $30 \%$ a $60 \%$. Nenhuma abordagem tera- pêutica, exceto a estratégia ventilatória (ventilação protetora-PEEP elevado e baixo volume corrente com a finalidade de abrir os pulmões e mantê-los abertos), apresentou impacto na sobrevida desta síndrome. Os maiores temas para a controvérsia são a manobra de recrutamento alveolar e o uso de elevados níveis de pressão positiva no final de expiração(PEEP).

\section{Comentário}

Em seu artigo, Carmen Valente Barbas ressalta a importância da tomografia computadorizada de tórax no diagnóstico e na definição da estratégia ventilatória a ser empregada, as evidências experimentais sobre a lesão associada à ventilação mecânica (hiperdistensão alveolar, abertura e colapso cíclicos dos alvéolos, biotrauma), e o papel da estratégia ventilatória protetora na lesão pulmonar em animais de experimentação. Enfatiza ainda a importância da manobra de recrutamento alveolar para tornar a distribuição de gás mais homogênea, descreve possíveis métodos de recrutamento alveolar, destacando de forma especial a manobra de recrutamento progressivo intermitente, e a importância da titulação da PEEP para a manutenção de uma ventilação mais homogênea. Tal manobra consiste na elevação progressiva dos níveis de PEEP $(25,30,35,40$ e 45 $\mathrm{CmH}_{2} \mathrm{O}$ ) até alcançar um plateau de pressão em torno de $60 \mathrm{cmH}_{2} \mathrm{O}$. Tais dados são muito relevantes, pois evidências cada vez mais fortes têm demonstrado o impacto desastroso, nos pulmões e em órgãos à distância (como os rins e os intestinos), da adoção de uma estratégia ventilatória inadequada.

Além disso, a autora projeta para o futuro a importância de métodos de monitorização pulmonar à beira do leito, como a tomografia de impedância elétrica, cujo objetivo consiste em analisar de forma contínua e não-invasiva a distribuição de gás nos pulmões durante a ventilação artificial. Este novo método poderá ser uma ferramenta extremamente útil na titulação fina da ventilação mecânica, contribuindo para a redução da mortalidade desta síndrome.

\section{Gustavo faissol Janot de Matos}

Referência

Barbas CSV. Lung recruitment maneuvers in acute respiratory distress syndrome and facilitating resolution. Crit Care Med 2003;3I(4):S265-S7I. 